

\title{
EL USO DE LA BIOTA LOCAL, COMO POSIBILIDAD PARA EL APRENDIZAJE DE LOS CONTENIDOS DE ENSEÑANZA DE LA ECOLOGÍA ${ }^{1}$
}

\section{Use of Local Biota as a Possibility to Learn the Contents of Ecology Teaching}

\section{O uso da biota local como possibilidade para a aprendizagem dos conteúdos de ensino da ecologia}

\author{
Ingrid Angélica Arias Santos ${ }^{2}$
}

Fecha de recepción: 22 de marzo de 2017

Fecha de aceptación: 13 de junio de 2017

\section{Resumen}

El presente trabajo, surge del proyecto de práctica pedagógica planteado como requisito de formación de la Universidad Pedagógica Nacional durante el año 2015. La investigación tuvo como objeto identificar los procesos de modificabilidad en el aprendizaje de contenidos de enseñanza de interacciones ecológicas tras la implementación de cuatro actividades, que pretendían diagnosticar, diseñar y evaluar, diversas estrategias didácticas que involucraran la biota local. Este proyecto se lleva a cabo en el Instituto Alberto Merani, con una población de 20 estudiantes del curso Proyectivo C (estudiantes en su último año escolar entre los 16 y 18 años). Se empleó como metodología el análisis de contenido para sistematizar y encontrar categorías que permitieran identificar los cambios producidos durante el estudio. Como principales conclusiones, se encuentra que la planificación y uso de actividades que involucren el contexto de los estudiantes puede favorecer la comprensión y transferencia de los contenidos abordados en clase, por otro lado los procesos de aprendizaje difieren significativamente entre estudiantes y pese al uso de estrategias diversas, en algunos estudiantes persisten las ideas previas adquiridas en su contexto social.

Palabras clave: Procesos de modificabilidad; biota local; interacciones ecológicas; desarrollo; aprendizaje.

\section{Abstract}

This paper is the result of the pedagogical practice project established as a graduation requirement of the National Pedagogical University in 2015. The object of investigation was to identify the processes of adjustability in the learning of teaching contents on ecological interaction after the implementation of four activities that aimed to diagnose, design and assess several didactic strategies involving the local biota. This project was carried out at the Alberto Merani Institute with 20 students from the "Proyectivo C" course (students between the ages of 16 and 18 who are in their last year of school). Content analysis was the methodology used to systematize and find categories, which made it possible to identify the changes occurred during the study. As main conclusions, it was found that the planning and use of activities involving the students' context may favor the understanding and transference

1 Este proyecto se lleva a cabo durante el ejercicio de práctica (un espacio academico obligatorio de práctica durante la formación de pregrado en Licenciatura en Biología, Universidad Pedagógica Nacional).

2 Licenciada en Biología - Candidata a magister en Educación- Universidad Pedagógica Nacional, Bogotá, Colombia, iaariass@upn.edu.co 
of the contents tackled in class. On the other hand, the learning processes differ significantly among students and, despite using several strategies, some students retain the previous ideas acquired from their social context.

Keywords: Adjustability processes; local biota; ecological interactions; development; learning.

\section{Resumo}

O presente documento surge do projeto de prática pedagógica formulado como requisito de formação da Universidade Pedagógica Nacional durante o ano 2015. A pesquisa teve como objetivo identificar os processos de modificabilidade na aprendizagem de conteúdos de ensino de interações ecológicas após a implementação de quatro atividades que procuravam diagnosticar, desenhare avaliar diversas estratégias que involucrassem a biota local. Este projeto efetua-se no Instituto Alberto Merani com uma população de 20 estudantes do curso "Proyectivo C" (estudantes no último ano escolar entre os 16 e os 18 anos). Como metodologia empregou-se a análise de conteúdo para sistematizar e encontrar categorias que permitissem identificar as mudanças produzidas durante 0 estudo. Como principais conclusões, encontra-se que o planejamento e o uso de atividades que envolvem o contexto dos estudantes pode favorecer a compreensão e a transferência dos conteúdos abordados na aula. Por outro lado, os processos de aprendizagem diferem significativamente entre estudantes e apesar do uso de diversas estratégias, em alguns estudantes prevalecem as ideias prévias adquiridas no seu contexto social.

Palavras chave: Processos de modificabilidade; biota local; interações ecológicas; desenvolvimento; aprendizagem. 


\section{Introducción}

Colombia se reconoce actualmente como un país con una de las mayores riquezas en flora y fauna. Su ubicación geográfica tan privilegiada ha permitido que se destaque a nivel mundial como uno de los países con la mayor cantidad de aves (66 especies), plantas (1500 especies), anfibios (367 especies), reptiles (115 especies), mamíferos (34 especies) y orquídeas (1543 especies), según datos del Sistema de Información sobre Biodiversidad de Colombia $(\mathrm{SIB})^{3}$. Sin embargo, y pese a la inmensa riqueza que nos representa, el desconocimiento de la misma y la falta de inclusión en los contenidos curriculares de la escuela ha hecho que día a día olvidemos la importancia en las relaciones sistémicas y holísticas que surgen de nuestra interacción diaria con la diversidad que nos rodea y nos privilegia frente a otras naciones.

Es de esta manera, y reconociendo este contexto, que la educación en Colombia no debería perder la enseñanza de temas relacionados con la comprensión y admiración de esta megadiversidad. Así, el estudio de la ecología, como un contenido relevante que ha sido avalado por la comunidad científica, se hace pertinente en las escuelas. Sin embargo, y como lo enuncian Bermudez y De Longhi, la enseñanza de estos contenidos deben considerar la "complejidad de variables relacionadas con las características del propio objeto de conocimiento, el enfoque curricular que se le dará y los aprendizajes que se esperan lograr" (2008, p. 275); de tal manera que las estrategias metodológicas y didácticas deben ser estudiadas y cobran sentido desde el mismo contexto e intencionalidad del mediador y los estudiantes, es en esta medida que se puede dar la apertura a otros campos del saber, como el científico.

Al respecto, conviene decir que la idea del conocimiento científico de la escuela "es una construcción social, producto de un trabajo humano, más que un conocimiento objetivo"; tal como lo menciona De Longhi (2000), al citar a Driver (1989), en este sentido la relación en los contenidos aprendidos se ven afectados por la estructura o esquema mental del que aprende y los diversos contextos de aprendizaje:

3 Datos oficiales de la SIB, Colombia. La SIB es una red de redes, encargada de facilitar herramientas para la integración, publicación y consulta de la información sobre biodiversidad (datos, metadatos, conjuntos de referencia y fichas de especies). https://www.sibcolombia.net/
Es así que cobran valor las ideas previas, las estrategias cognitivas, metacognitivas y los propósitos e intereses de los alumnos; importa el sentido de cada situación de enseñanzayaprendizajepara cada individuo, grupo y momento. Se retoma la dimensión interaccionista del aprendizaje y se tiene en cuenta que la construcción del conocimiento depende de un proceso de negociación social. (De Longhi, 2000, p. 201).

A partir de lo anterior, la enseñanza de interacciones ecológicas sin lugar a dudas representa todo un reto para maestros y estudiantes, debido a que genera un pensamiento crítico y revolucionario que nos incluye en la trama de la vida y por tanto como parte de la evolución biológica (Castro, 2015). Este tipo de pensamiento permite generar diversas posturas sobre el mundo que nos rodea. Al respecto, conviene recordar que "la evolución nos dice que existimos por una serie de acontecimientos pasados que no difieren en su naturaleza de los acontecimientos y procesos que han dado lugar a los millones de organismos que nos rodean" (Dobzhanshy, Ayala, Stebbins y Valentine, 2009). Para poder comprender el sentido y las relaciones que vinculan nuestra especie con las demás, se hace pertinente estudiar las interacciones que se dan entre los diversos organismos y el ambiente que ha cambiado a lo largo del tiempo.

Como apunta Dobzhansky (1970), en un mundo que puede tener de cuatro a cinco millones de tipos distintos de organismos, en un amplio número de hábitats, la variedad de posibles interacciones entre las poblaciones y su medio ambiente resulta enorme. El alcance de estas interacciones es también muy diverso, desde las profundidades del océano, en laderas, desiertos en la sabana, tundras y bosques húmedos por tomar algunos ejemplos (Dobzhansky, Ayala, Stebbins y Valentine, 2009).

Sin embargo, las formas en las que se desarrolla el discurso de esta temática puede favorecer la comprensión de la misma o generar obstáculos en su comprensión (De Longhi, 2000). Es en medio de esta complejidad que el papel del maestro cambia y se reconoce su responsabilidad profesional, desde la construcción conjunta con el estudiante, permitiéndole crear en su mente respuestas alternativas de mundo, $y$ haciendo de este aprendizaje algo significativo, que él emplee y le permita reconfigurar sus formas de comprender diferentes fenómenos que ocurren; es aquí donde el papel del mediador toma sentido: 
Como su mismo nombre lo indica, un intermediario, un amplificador, un adaptador, un organizador y un diseñador de procesos formativos. Con lo que el ser diseñador le implica conocer cuánto contribuye a la auténtica construcción de la obra más compleja que podemos acometer, como es la formación de una persona. (Tebar, 2009, p. 20).

Dentro de los aportes de este mediador, se reconoce su papel activo dentro del discurso y las formas en las que desarrolla sus didácticas, en este contexto se reconocen los aportes de Jean Piaget y Vygotsky (1979), "desde la psicología, y fundamenta el lugar que ocupa la mediación que provoca el lenguaje en el aprendizaje" (De Longhi, 2000). Es por esto que se hace importante en el proceso de aprendizaje reconocer la zona de desarrollo real del niño y la zona de desarrollo próxima del mismo; es decir, identificar la distancia entre el conocimiento existente en el niño, en su proceso inicial y lo que se pretende que él aprenda con ayuda del otro (De Longhi, 2000), haciendo uso de ideas previas, de tal forma que al cierre del proceso académico se logren producir rectificaciones, jerarquizaciones o ampliaciones conceptuales de las mismas (Bermudez y De Longhi, 2008).

Al reconocer que el sujeto crea unas concepciones a lo largo de su vida y las emplea para dar respuesta a los fenómenos que observa, los procesos de modificabilidad cognitiva estructural toman sentido. En primera medida, es importante destacar al autor de este postulado, R. Feuerstein, que postula esta forma de comprender los procesos de re-estructuración, viendo al ser humano como un sistema abierto, que es susceptible de construirse activamente en sociedad. En esta medida, Feuerstein indica que "la esencia de la inteligencia, no radica en el producto mensurable (que se puede medir), sino en la construcción del individuo" (Citado por Tebar, 2009, p. 19), por tanto, el individuo tiene capacidad para usar experiencias adquiridas previamente, para ajustarse a nuevas situaciones De esta manera, uno de los escenarios donde pueden darse estos procesos de modificabilidad es la escuela.

Sin embargo, y para que esto ocurra, el mediador debe diseñar propuestas que presenten intencionalidad, trascendencia y significado (Tebar, 2009), una de las estrategias que se han diseñado para hacer trascendente el conocimiento en biología, se propone desde el estudio de especies vivas, con el propósito de que el estudiante logre hacer de su conocimiento algo concreto y cercano que lo vincule y le permita ver que lo que aprende en la escuela tiene sentido en su vida diaria. Tal y como lo menciona Matthews (1994).
Valdría la pena resaltar que el trabajo que se ha llevado con lepidópteros, especialmente las mariposas, ha sido una estrategia positiva en la comprensión de interacciones ecológicas, puesto que dicha especie ha sido exaltada en contextos científicos y artísticos desde la antigüedad (Torres, 2013). Las mariposas cumplen un papel muy importante en el mantenimiento y regulación de los ecosistemas, ayudan en la polinización, fuente de alimento a otros organismos, y se involucran en complejas interacciones ecológicas y coevolutivas, además de ser utilizadas como un instrumento para educar debido a su gran potencial de brindar experiencias positivas en la enseñanza de la biología, razón por la cual se están desarrollando, aplicando, evaluando y divulgando métodos y materiales novedosos, potencialmente productivos para los profesores y sus estudiantes alrededor del estudio de los insectos (Torres y García, 2011). De esta manera, los maestros debemos aprender a hablar en términos de experiencias profesionales, como dice Tardif, "El maestro 'no piensa sólo con la cabeza', sino 'con la vida', con lo que ha sido, con lo que ha vivido, con lo que ha acumulado en términos de experiencia vital, en términos de bagaje de certezas" (2003, p. 93).

A partir del panorama anterior, el presente informe muestra los resultados que surgen de una serie de actividades y propuestas didácticas que se desarrollan con los estudiantes del ciclo Proyectivo C, del Instituto Alberto Merani; en el Seminario de Ecología (uno de los espacios académicos, que cursan los estudiantes en el cierre de su proceso escolar). Reconociendo el contexto, la zona de desarrollo de los estudiantes (De Zubiría et al., 2009) y las intenciones generales del curso, se toma como tema nuclear e integrador las interacciones ecológicas, además se hace uso de ejemplos locales, analogías y la observación del ciclo de vida de la especie Leptophobia aripa, para comprender el tema de interacciones ecológicas.

\section{Metodología}

De acuerdo con lo planteado, el desarrollo del presente proyecto usó como metodología de investigación el enfoque de corte cualitativo interpretativo, del que ha señalado Cerda: "Utiliza múltiples fuentes, métodos e investigadores para estudiar un solo problema o tema, los cuales convergen en torno a un punto central del estudio" (Cerda, 1991, p. 48). Conviene mencionar que se tomaron varios instrumentos para la recolección de datos, la consulta, la revisión y el análisis de referencias teóricas asociadas a la temática planteada (interacciones ecológicas), adicionalmente, se realizó un registro de situaciones propias 
del ambiente escolar que contribuyeron a reconocer las concepciones de los estudiantes acerca de las interacciones ecológicas, partiendo inicialmente de una prueba diagnóstica que se contrastó a lo largo de las actividades, para poder evaluar sus modificaciones con el uso de estrategias didácticas que permitieron orientar las acciones pedagógicas en pro de un aprendizaje contextual y sistémico.

El proyecto se realizó con 20 estudiantes del ciclo Proyectivo $C$, del Instituto Alberto Merani, estudiantes entrelos 16y los 18 años; lasactividadesque se plantearon, se diseñaron atendiendo la zona de desarrollo real y próxima de los estudiantes, los propósitos de pedagogía dialogante y la actividad rectora del ciclo (De Zubiría et al., 2009) (entendiendo este como el núcleo integrador y nuclear que debe lograrse con el proceso académico); que para este ciclo es

\begin{abstract}
Debatir con el grupo de pares y definir el proyecto de vida[...] Un joven, se podría pensar, loqueesen tanto hace parte de un grupoy debate con él. Es un ciclo para definir el proyecto de vida, con énfasis en la dimensión socio afectiva y en la identidad. (De Zubiría et al., 2009).
\end{abstract}

Se categorizaron e interpretaron las respuestas de cuatro ejercicios que fueron el foco de la presente investigación (estos se presentan con más detalle en los resultados) y se desarrollaron a lo largo del primer semestre del año 2015, mediante observación participante y no participante. Los criterios que se utilizaron para llevar a cabo esta sistematización, se dieron a través del análisis de contenido debido a que, esta técnica permitió revisar y analizar las respuestas de manera objetiva, sistemática y cualitativa, para ubicar las categorías comunes (Hernández, Fernández y Baptista, 2006).

Adicionalmente, se siguieron las pautas que recomienda Chavez (1986), al ubicar en cada una de las respuestas obtenidas núcleos de referencia, que corresponden a los aspectos más importantes de los resultados obtenidos y que permitieron evidenciar el impacto de las actividades; de tal forma que se logró registrar el cambio en los discursos y la estructura argumentativa de los estudiantes a lo largo del desarrollo del presente proyecto.

Cabe resaltar que el tratamiento de los resultados obtenidos parte de una categorización deductiva, de esta manera, y de acuerdo con lo que plantean Bonilla y Rodriguez (2005), la categoría que se establece es interacciones ecológicas, esta se encontraba definida a partir del marco teórico consultado previamente y los instrumentos aplicados tenían una intencionalidad clara que pretendía conocer la comprensión de esta temática en los estudiantes y la modificabilidad de preconcepciones en torno a la misma.

\section{Descripción breve de los instrumentos}

Instrumento 1. El día 27 de marzo, como inicio de la propuesta de investigación, se realizó la aplicación de la prueba diagnóstica. Esta prueba se realizó de forma escrita, a 20 estudiantes del curso proyectivo C, e incluía imágenes y preguntas que implicaban la temática de interacciones ecológicas de forma implícita y explicita. Se empleó el método de categorización deductivo, de esta manera, a partir de las respuestas de los estudiantes, se ubican núcleos de referencia, que permitieron establecer categorías de análisis que acogieron varias de las respuestas de los estudiantes para ser sistematizadas y relacionadas estadísticamente, esto con el fin de reconocer tendencias generales entre los estudiantes y sus concepciones en torno a interacciones ecológicas.

Instrumento 2. El día 15 de abril de 2015 se presentó a los estudiantes el tema de interacciones ecológicas. Como introducción al tema, la maestra en formación e investigadora lee en voz alta uno de los capítulos de Tropical Nature: "La cresta de Jerry". Esta historia narra la vivencia de un investigador que en medio del Amazonas es picado por un moscardón, su experiencia representa de forma vívida la interacción parasitaria.

La actividad tiene continuidad el día 20 deabril. En esta fecha, se presenta a los estudiantes la temática de interacciones por medio de material audiovisual. A partir de la introducción de este tema se presenta a los estudiantes una guía sobre el ciclo de vida de las mariposas, guíaque incluye para su lectura las estrategias de supervivencia (Ky R). Esto con la intención de ampliar los contenidos y aclarar dudas del proceso.

Instrumento 3. El día 11 de mayo de 2015, se organizaron unas mesas de trabajo para establecer un debate entre los estudiantes sobre el tema de protección ambiental y causas de calentamiento global; en medio de estas discusiones, emergen cuestiones que responden a la necesidad de protección de la biodiversidad. De esta manera se expone una problemática mundial que se relaciona con el tema de interacciones ecológicas y corresponde a la extinción masiva de abejas, esto con la intención de profundizar en el tema de interacciones ecológicas y cuestionar su posición frente a esta problemática. Se comparte con los estudiantes la siguiente cita: "Si la abeja desapareciera de la superficie del globo, al hombre solo le quedarían cuatro años de vida: sin abejas, no

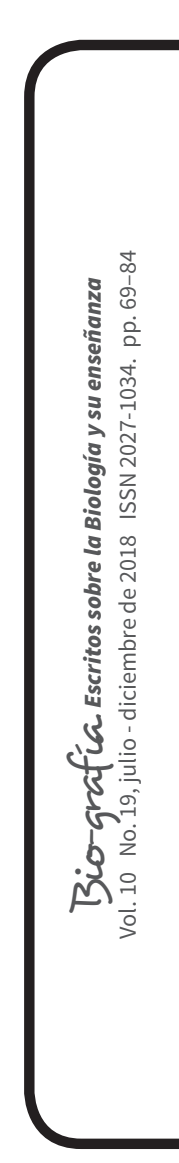


hay polinización, ni hierba, ni animales, ni hombres" (Albert Einstein).

A partir de esta cita se realizan dos preguntas orientadoras: 1) ¿por qué cree usted que pueden estar desapareciendo las abejas? 2) ¿Qué consecuencias traería la desaparición de estos organismos para la ecología? Estas preguntas se realizan con el propósito de reconocer si el estudiante aplica lo visto en clase, en situaciones reales y si hace una transferencia de los conceptos empleados en clase, prediciendo las causas y el fenómeno de la situación descrita.

Instrumento 4. El día 29 de mayo, a modo de cierre del ejercicio de investigación, se aplica la prueba diagnóstica inicial, esto con el fin de comprobar si se dieron procesos de modificabilidad en los estudiantes, con la aplicación de las actividades y los ejemplos socializados en clase. A partir de las respuestas de los estudiantes, se evidencian las siguientes tendencias: los estudiantes coinciden en que el fenómeno que se presenta es depredación, en algunos casos usan la descripción de la imagen, depredación y alimentación como factor determinante en la supervivencia, es necesaria la disputa entre los organismos, los estudiantes reconocen que ven diversas interacciones ecológicas y las relacionan con ejemplos cotidianos.

\section{Resultados}

A partir de la aplicación del instrumento 1 y de las respuestas de los estudiantes se realizan las gráficas que recogen la frecuencia de los datos obtenidos.

Instrumento 1. Prueba diagnóstica.

\begin{tabular}{|c|c|c|}
\hline Descriptor. Preguntas & Evidencia & Categoría \\
\hline $\begin{array}{l}\text { ¿De las imágenes que se } \\
\text { muestran a continuación, } \\
\text { explique qué observa y con qué } \\
\text { fenómeno lo asocia? }\end{array}$ & 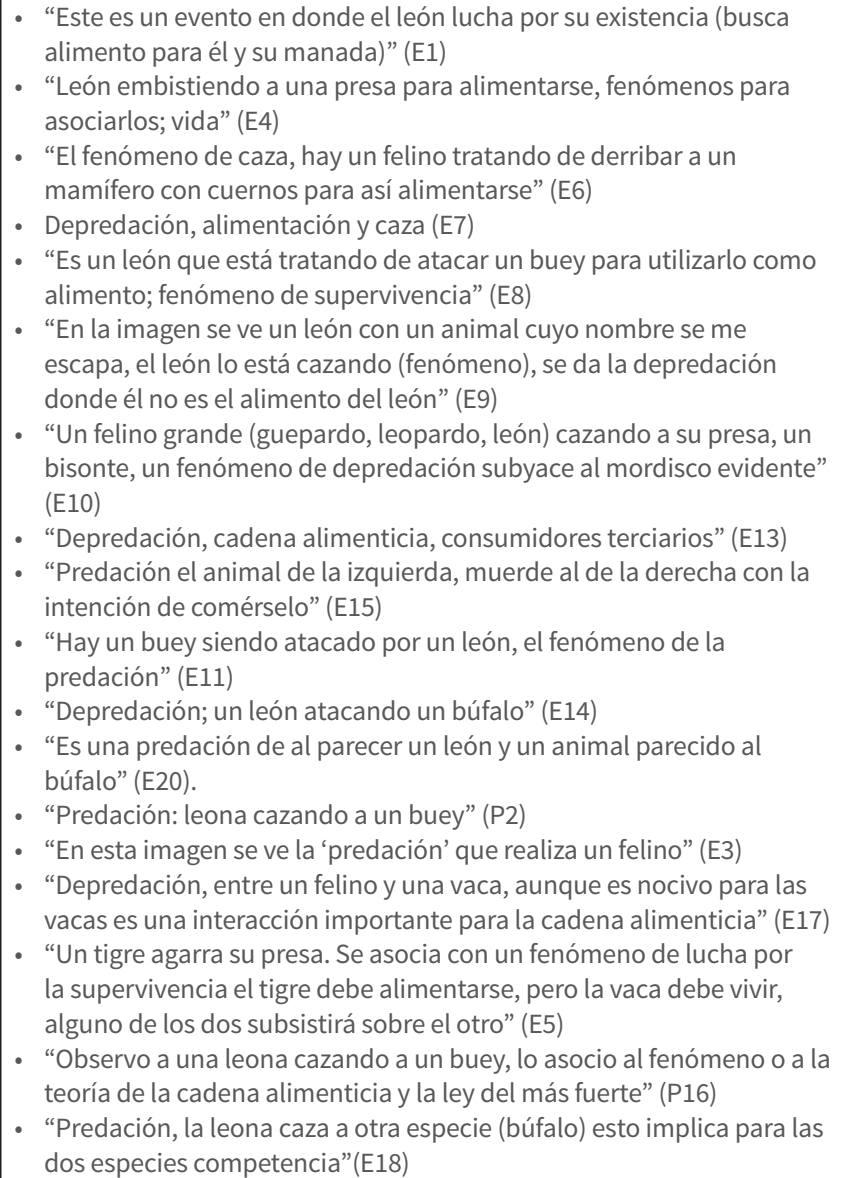 & $\begin{array}{l}\text { - Alimentación, como proceso } \\
\text { inherente de supervivencia. } \\
\text { Se reconoce el proceso de } \\
\text { depredación y se asocia con } \\
\text { procesos de alimentación y } \\
\text { cadena alimenticia. } \\
\text { - Lucha por la existencia; la ley } \\
\text { del más fuerte. } \\
\text { - Relación de dos } \\
\text { interacciones: depredación y } \\
\text { competencia. }\end{array}$ \\
\hline
\end{tabular}




\begin{tabular}{|c|c|c|}
\hline Descriptor. Preguntas & Evidencia & Categoría \\
\hline & 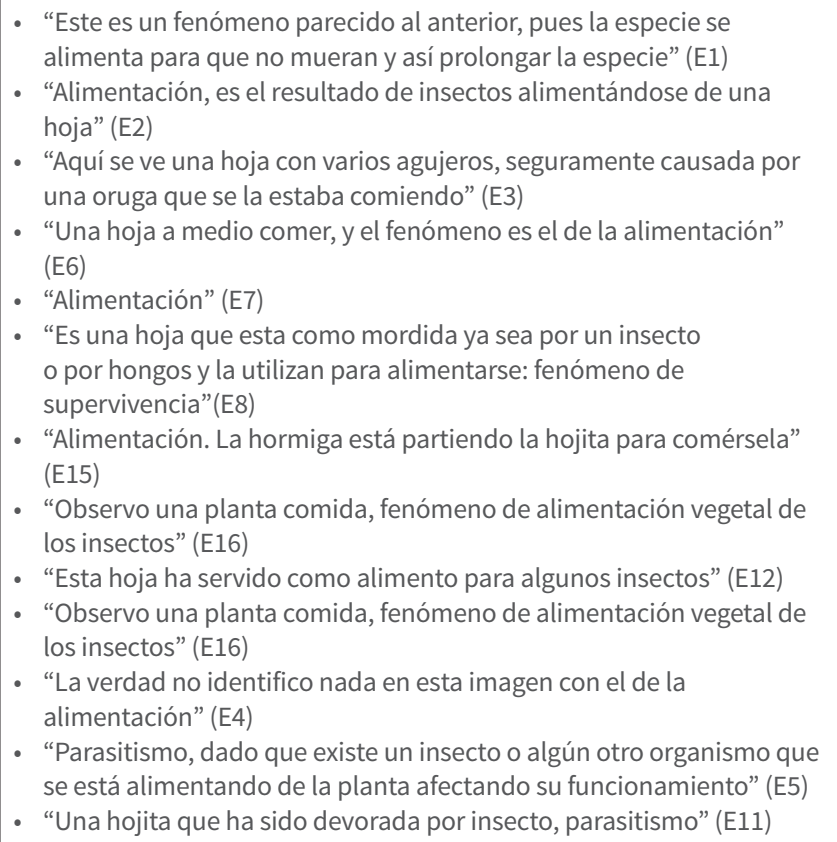 & $\begin{array}{l}\text { - Alimentación y } \\
\text { supervivencia. } \\
\text { - No se ve la relación entre } \\
\text { una hoja con agujeros y el } \\
\text { fenómeno de alimentación. } \\
\text { - Parasitismo. }\end{array}$ \\
\hline VSTANOS EN TAMOCRAZYNET \& & $\begin{array}{l}\text { - "No sé qué fenómeno sería este grano sobre la nuca de un humano" } \\
\text { - "E1) } \\
\text {-Aquí se ve un hombre con una erupción enorme en el cuello que } \\
\text { pudo ser causada por el crecimiento de una larva entre su piel o un } \\
\text { caso exagerado de acné" (E3) } \\
\text { - "Un grano ha aparecido en la nuca del joven más desafortunado del } \\
\text { - "Uglo xxl, un fenómeno más conocido como adolescencia" (E10) } \\
\text { "Una pústula que se da por la infección de un poro generando pus por } \\
\text { - "Observo un grano gigante una gran acumulación focalizada de } \\
\text { lípidos y grasas" (E14) } \\
\text { - "Algún tipo de desequilibrio en las relaciones del cuerpo" (E15) } \\
\text { - "Un grano o lípidos (grasa) en la espalda de una persona" (E20) } \\
\text { una infección o una enfermedad que ha penetrado el organismo del } \\
\text { individuo y lo está perjudicando" (E5) } \\
\text { - "Se observa una reacción probablemente alérgica a una picadura de } \\
\text { un insecto" (E6) }\end{array}$ & $\begin{array}{l}\text { - Se interpreta como un factor } \\
\text { asociado a factores que } \\
\text { han experimentado en su } \\
\text { adolescencia como el acné. } \\
\text { - Se encuentra asociado a } \\
\text { fenómenos de infección. }\end{array}$ \\
\hline $\begin{array}{l}\text { Pregunta } 2 \\
\text { De las aves que se muestran a } \\
\text { continuación marque con una } \\
\text { "X" el grupo que considera tiene } \\
\text { una ventaja mayor, a la hora } \\
\text { de obtener su alimento, sobre } \\
\text { las otras y mencione por qué la } \\
\text { selecciona. }\end{array}$ & $\begin{array}{l}\text { - "Las aves grandes tienen ventaja sobre las chiquitas" (E1) } \\
\text { - "Las aves grandes, la forma y la longitud de su pico seguramente les } \\
\text { - "Las aves grandes, parece ser que tienen un pico más largo que las de } \\
\text { los otros grupos, ventaja importante para alimentarse mejor" (E4) } \\
\text { - "De mayor tamaño, si bien los picos son cortos el tipo de alimento } \\
\text { es distinto a las de los picos alargados y gracias a las ventajas físicas, } \\
\text { será más fácil su alimentación" (E6) } \\
\text { - "Las aves grandes; las características físicas de esta ave llevan a } \\
\text { pensar que tiene más posibilidades de supervivencia que las otras } \\
\text { una de ellas, el pico alargado y puntiagudo" (E10) } \\
\text { - "Mayor tamaño y picos más grandes" (E15) } \\
\text { "Los pájaros con cabezas grandes; son pájaros que poseen cabezas } \\
\text { mejor" (E20) } \\
\text { - "Las aves pequeñas de picos largos, pues estas podrían alimentarse } \\
\text { de flores e insectos ya que no están totalmente especializados” (E7) } \\
\text { "Las aves pequeñas de picos largos este grupo tiene mayor ventaja } \\
\text { debido a que parecen ser aves más rápidas y un pico que es grande y } \\
\text { a la vez puntiagudo" (E8) } \\
\text { - "Aves de picos largos, se alimenta de flores y no tiene tanta } \\
\text { competencia” (E19) } \\
\text { "Aves de picos medianos y largos, tal vez porque son aves } \\
\text { depredadoras con un nivel de peligro o predación por parte de otras } \\
\text { especies, adicional de habilidades físicas importantes" (E16) } \\
\text { - No saben (E5, E2) }\end{array}$ & $\begin{array}{l}\text { - Se asocia el tamaño grande } \\
\text { con mayor ventaja para } \\
\text { obtener alimento desde la } \\
\text { interacción. } \\
\text { - Aves pequeñas de picos } \\
\text { largos, por tener menos } \\
\text { competidores. } \\
\text { - No reconocen una respuesta. }\end{array}$ \\
\hline
\end{tabular}




\begin{tabular}{|c|c|c|}
\hline Descriptor. Preguntas & Evidencia & Categoría \\
\hline \multirow[t]{2}{*}{$\begin{array}{l}\text { Indique que entiende por } \\
\text { competencia ecológica y } \\
\text { mencione qué organismos } \\
\text { relaciona más fácilmente con } \\
\text { el tema }\end{array}$} & $\begin{array}{l}\text { - "Es la competencia entre especies para sobrevivir, por ejemplo una } \\
\text { caza de los felinos" (E16) } \\
\text { - "La competencia ecológica hace alusión a la capacidad o capacidades } \\
\text { de cualquier organismo vivo que le permite sobrevivir. Los seres } \\
\text { humanos y los animales" (E20) } \\
\text { - "La competencia ecológica es una relación biológica que condiciona } \\
\text { la supervivencia poniendo en juego elementos como 1) espacio 2) } \\
\text { alimento 3) reproducción 4) otros" (E3) } \\
\text { - "Entiendo por competencia ecológica una relación de lucha } \\
\text { o contienda entre organismos por dominar y subsistir en un } \\
\text { ecosistema. Esta competencia no solo se da entre individuos, sino } \\
\text { también entre organismos y factores abióticos" (E15) }\end{array}$ & $\begin{array}{l}\text { - Como factor asociado a la } \\
\text { supervivencia. }\end{array}$ \\
\hline & $\begin{array}{l}\text { - "Es una relación de tensión de supervivencia en la que dos o más } \\
\text { organismos están implicados. Una ballena y el krill; por ejemplo" }\end{array}$ & $\begin{array}{l}\text { Elemento de supervivencia. } \\
\text { Sin embargo, lo relacionan } \\
\text { con organismos foráneos (no } \\
\text { tienen en cuenta la fauna } \\
\text { local). }\end{array}$ \\
\hline $\begin{array}{l}\text { ¿En el lugar en el que } \\
\text { usted habita es posible ver } \\
\text { interacciones ecológicas? } \\
\text { ¿Cómo las reconoce? } \\
\text { Argumente su respuesta y } \\
\text { brinde algunos ejemplos. }\end{array}$ & $\begin{array}{l}\text { - "Aunque claramente las hay, no los veo o reconozco pues no es algo } \\
\text { que en la cotidianidad de una ciudad se observe" (E16) } \\
\text { - "No sé" (E17) } \\
\text { - "En todas partes de nuestro alrededor hay interacciones ecológicas; } \\
\text { por ejemplo, es la fotosíntesis" (E1) } \\
\text { - "A veces se ven aves que se alimentan de lombrices" (E12) } \\
\text { el sistema deja de estar en equilibrio" (E15) }\end{array}$ & $\begin{array}{l}\text { - No reconoce interacciones } \\
\text { ecológicas. } \\
\text { - Se presenta reconocen } \\
\text { fenómenos como la } \\
\text { fotosíntesis como una } \\
\text { interacción ecológica. } \\
\text { - Se emplean eventos } \\
\text { cotidianos para dar } \\
\text { explicación al fenómeno. }\end{array}$ \\
\hline
\end{tabular}

Fuente: elaboración propia

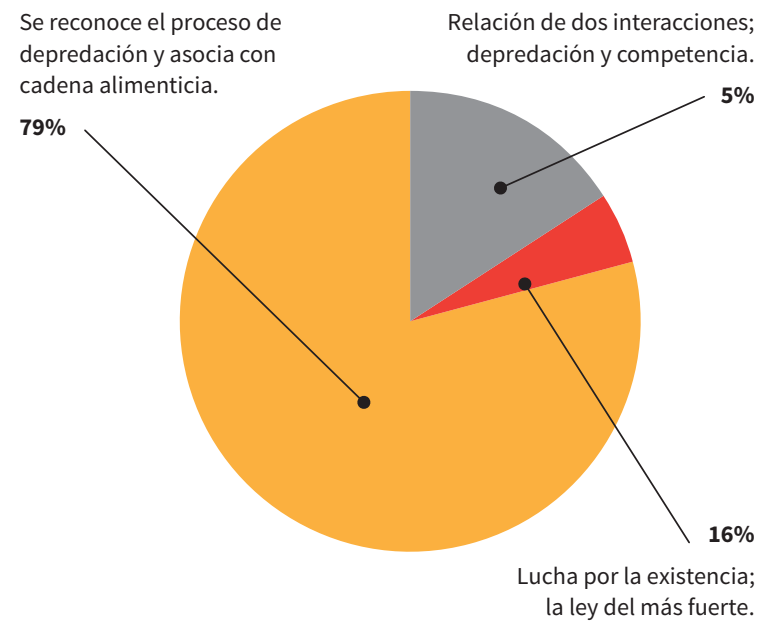

Grafico 1. Descripción de la imagen pregunta 1

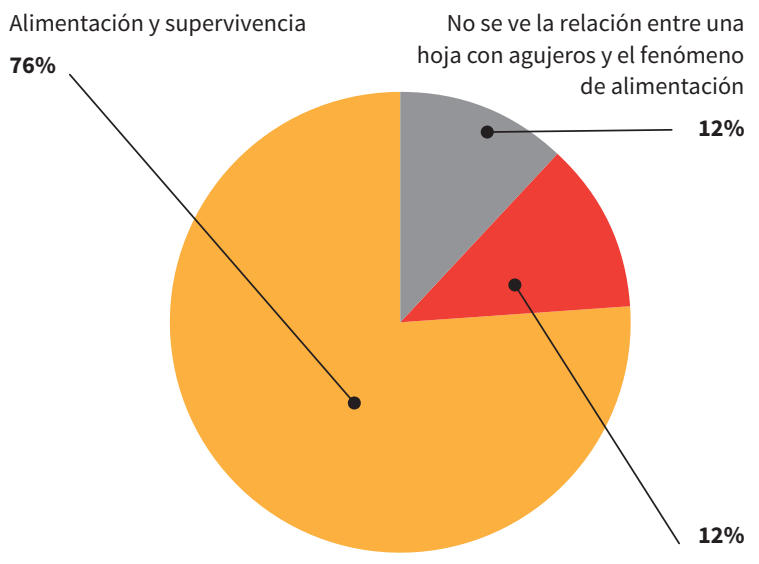

Parasitismo

Grafico 2. Descripción de la imagen pregunta 1 


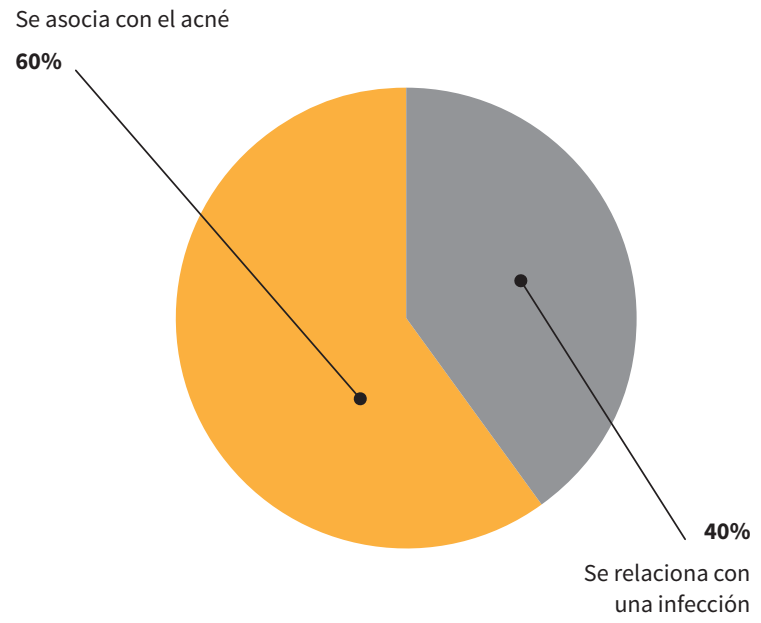

Grafico 3. Descripción de la imagen pregunta 1

En este primer ejercicio cabe resaltar que los estudiantes, en su mayoría se quedan en la descripción de la imagen y responden de acuerdo a vivencias personales. Es interesante cómo el estudiante reconoce en la primera imagen (una leona atacando a un búfalo) un proceso de depredación, pero noasocian el mismo fenómeno alverunahojacomida. Porotrolado, el porcentaje de estudiantes que relacionan el fenómeno de parasitismo con una hoja comida puede implicar lógicas de pensamiento un poco más sistémico, porque pueden estar asociando que el consumo del depredador es parcial sobre el árbol.

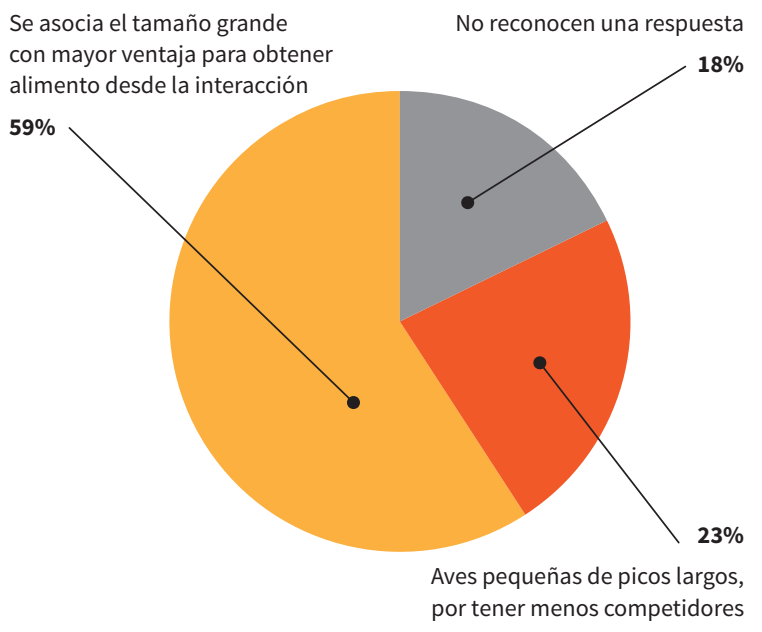

Grafico 4. Pregunta 2
En este ejercicio los estudiantes, en su mayoría, consideran que las aves más grandes aventajan a las más pequeñas. Esto puede estar asociado a vivencias propias o al estudio previo de biología de comportamiento humano, donde se relaciona un mayor tamaño con ventajas adaptativas sobre otros individuos de la especie o sobre otras especies, porque de una u otra manera se muestra dominancia sobre las demás; pero, se deja de lado que de acuerdo con las condiciones ambientales y adaptaciones al medio, cada especie puede tener unas características diferentes por sus requerimientos alimenticios o hábitos y no siempre los individuos más grandes tienen ventajas adaptativas sobre otros.

Pregunta 3. Indique qué entiende por competencia ecológica y mencione qué organwismos relaciona más fácilmente con el tema

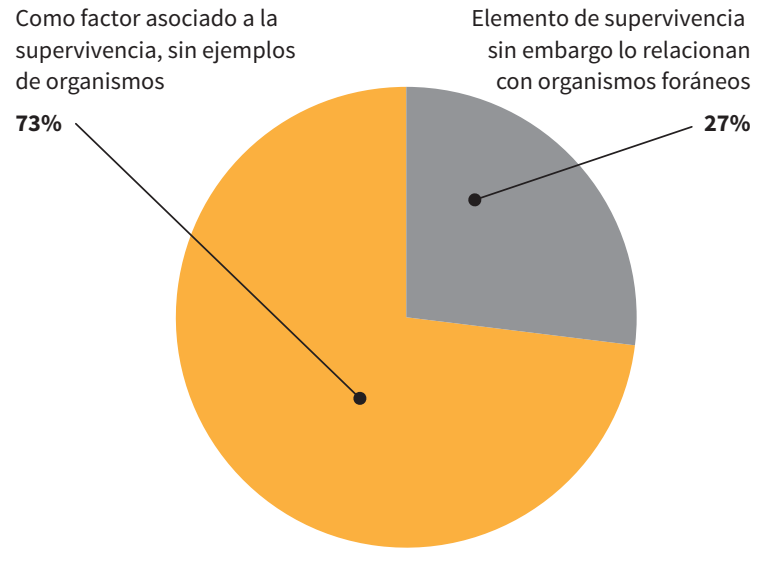

Grafico 5. Pregunta 3

De acuerdo con lo que responden los estudiantes, cabe resaltar que este es un fenómeno determinante en la supervivencia de una especie sobre otra. Sin embargo, la mayoría de ellos no describe un ejemplo de este fenómeno, y tan solo el $27 \%$ de ellos describe un ejemplo, pero no relacionan fauna local, por el contrario se remiten a ejemplos de sabanas africanas o especies marinas (algo con lo que ellos no conviven diariamente, pero que pueden observar en documentales o viajes que hayan realizado). 
Se presenta reconocen fenómenos como la fotosíntesis como una

interacción ecológica

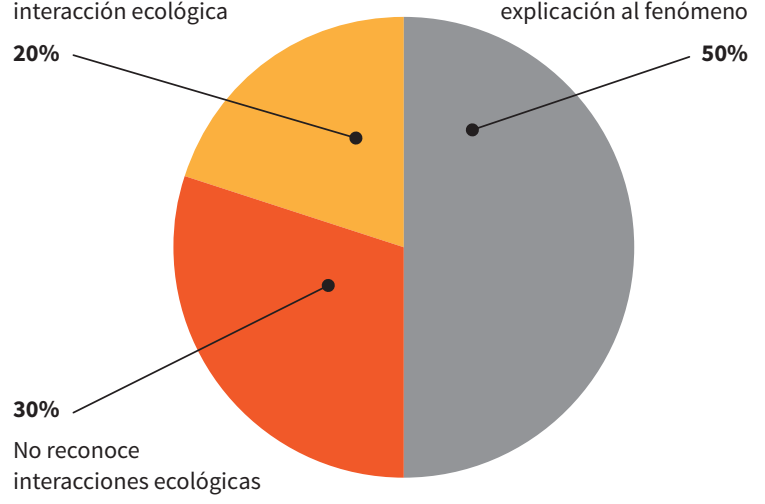

Grafico 6. Pregunta 6
Se emplean eventos cotidianos para dar explicación al fenómeno

\section{Instrumento 2. Taller en clase}

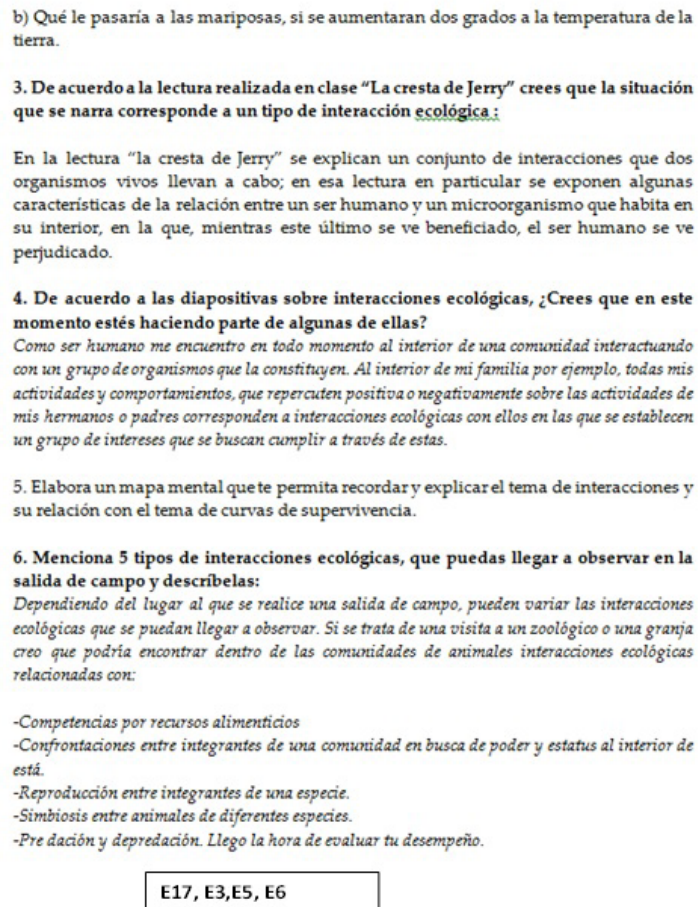

En la lectura "la cresta de Jerry" se explican un conjunto de interacciones que dos organismos vivos llevan a cabo; en esa lectura en particular se exponen algunas caracteristicas de la relación entre un ser humano y un microorganismo que habita en su interior, en la que, mientras este último se ve beneficiado, el ser humano se ve perjudicado.

4. De acuerdo a las diapositivas sobre interacciones ecológicas, ¿Crees que en este momento estés haciendo parte de algunas de ellas?

Como ser humano me encuentro en todo momento al interior de una comuridad interactuando con unt grupo de organismos que la constituyen. Al interior de mi familia por ejemplo, todas mis actividadesy comportamientos, que reperouter positiva onegativamente sobre las actividades de

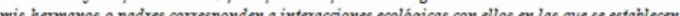
wen gnepo de intereses que se buscan cumplir a través de estas.

5. Elabora un mapa mental que te permita recordary explicar el tema de interacciones $y$ su relación con el tema de curvas de supervivencia.

6. Menciona 5 tipos de interacciones ecológicas, que puedas llegar a observar en la salida de campo y describelas:

Dependiendo del lugar al que se realice una salida de campo, pueden variar las interacciones ecológicas que se puedan llegar a observar. Si se trata de una visita a un zoológico o una granja creo que podria encontrar dentro de las comuridades de animales interacciones ecológicas relacionadas con:

-Competencias por recursos alimenticios

- Confrontaciones entre integrantes de una comurridad en busca de poder y estatus al interior de - Contá.

Reproducción entre integrantes de una especie.

-Simbiosis entre animales de diferentes especies.

-Pre dación y depredación. Llego la hora de evaluar tu desempeño.

E17, E3,E5, E6

La mitad de los estudiantes que presentan el ejercicio reconocen que se presentan interacciones ecológicas y las asocian a ejemplos como "un ave consumiendo un gusano", sin embargo, no profundizan en lo que implica esta interacción o qué tipo de interacción podrían estar observando. Por otro lado, es curioso ver que algunos estudiantes relacionen otros fenómenoscomolafotosíntesis como una interacción ecológica, debido a que, aunque este proceso es importante para la fabricación de alimento, no corresponde a una interacción ecológica, autores como Kricher definen y relacionan este proceso con productividad primaria: "Describir la cantidad de radiación solar, transformada por las plantas en moléculas complejas, como los azúcares”(Kricher, 2010, p. 40).

\begin{abstract}
1. ¿Sabes que es supervivencia? Explica brevemente este proceso y en donde lo encuentras.

La supervivencia podría definirse como el conjunto de comportamiento y características de los organismos vivos que les permiten satisfacer sus necesidades biológicas al interior de un determinado lapso de tiempo. La supervivencia esta presente en cualquier comunidad o contexto dentro del cual convivan organismos vivos, y en esa medida es posible encontrarla en cuasi cualquier parte de la superficie terrestre y acuática de nuestro plantea.
\end{abstract}

2. ¿Cuál es el ciclo de vida de los seres vivos que conoces?

Los seres humanos son organismos vivos sobre los que, a lo largo de su vida, ( $y$ a la igual que, el resto de organismos del planeta) es posible identificar un conjunto de caracteristicas y deetapas que marcan su desarrollo a nivel biológico. El ciclo de vida de los humanos, de la manera en que yo lo entiendo, puede dividirse, y comprenderse, a través de cuatro principales etapas de su desarrollo a nivel fisico y mental, que son: La infancia, la juventud, la "adultez", y la vejes. En cada una de estas etapas, el ser humano, mantiene las caracteristicas que lo constituyen como sistema vivo, pero va cambiando algunas de las caracteristicas que determinan su desarrollo fisico y mental y que en consecuencia determinan también el rol que este cumple como individuo al interior de una comunidad.

3. ¿Crees que en la naturaleza se presente competencia?:

La competencia es una característica intrinca de casi cualquier individuo que convive el interior de una comanidad. Todos los seres vivos, y mas claramente los seres hamanos (que creo que palican la estrategia $K$ ), compiten entre sí, no solo para la obtención de recursos que les permitan satisfacer sus necesidades vitales, sino también para la adquisición de un estatus o roles específicos al interior de una comunidad.

E15, E9,E11, E13 

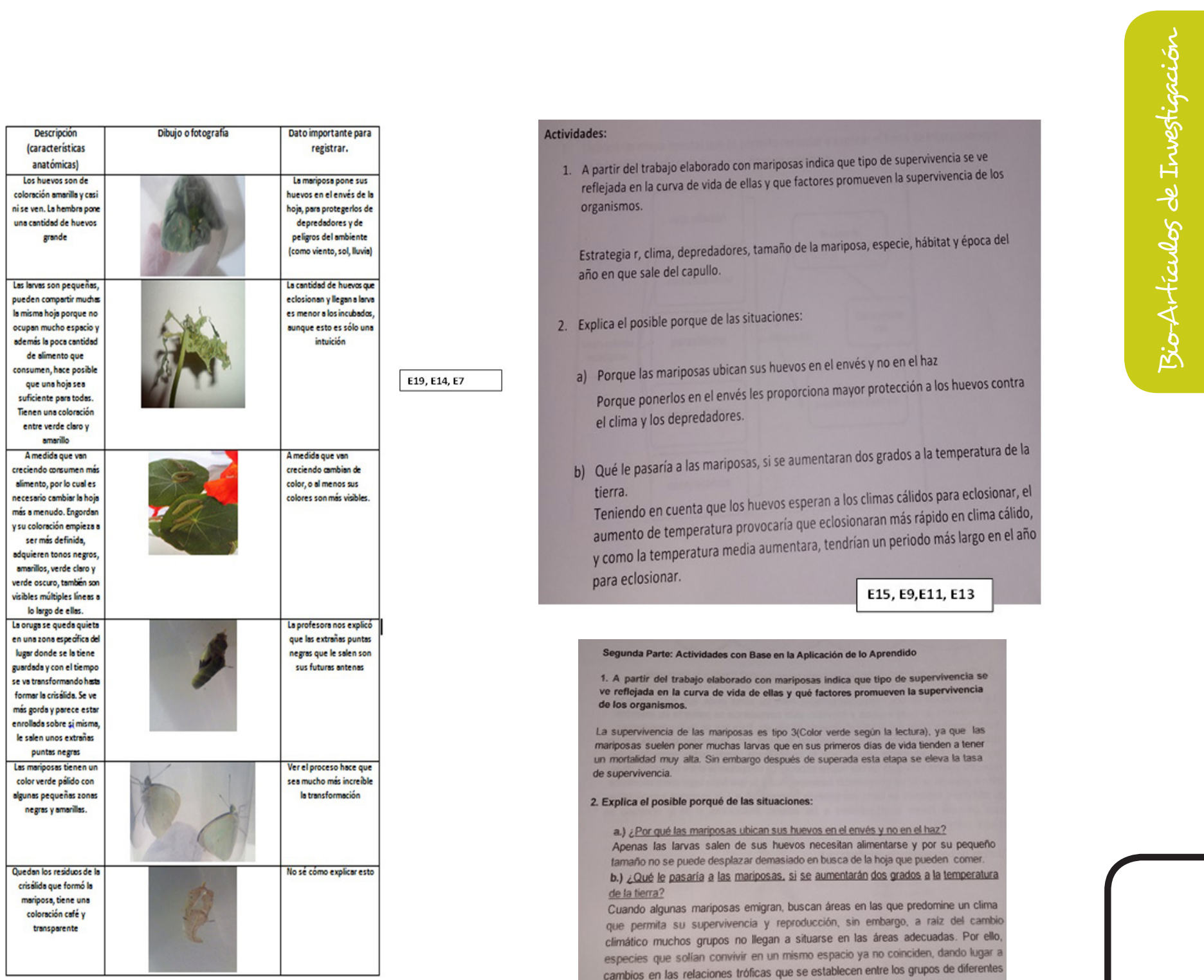


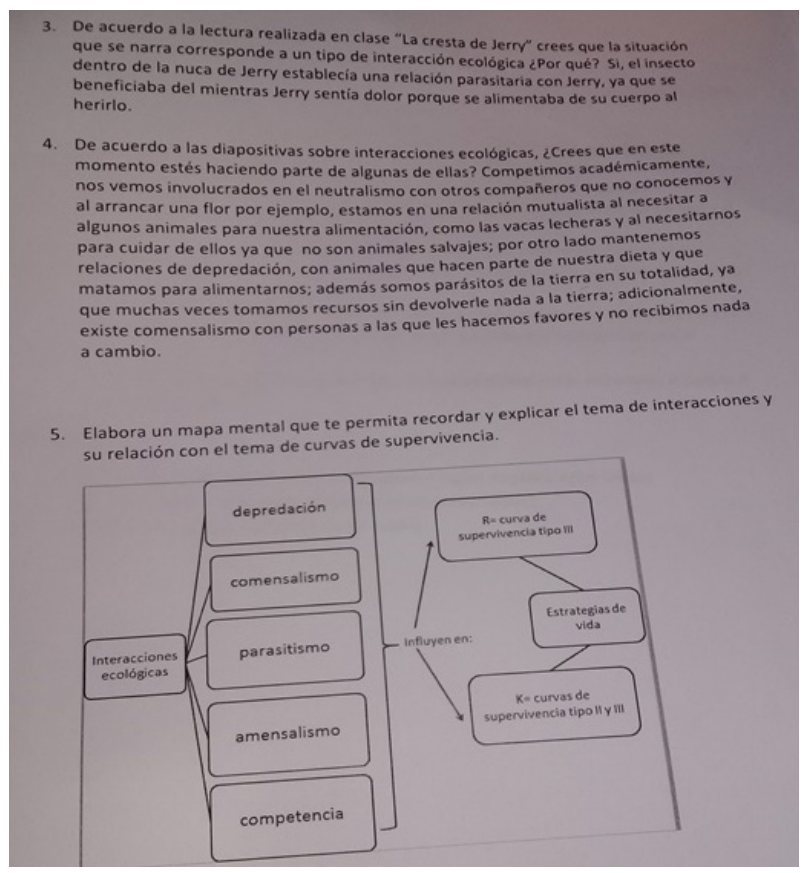

Con respecto a las respuestas de este taller, se logra evidenciar que los estudiantes profundizan en las mismas, además entran a considerar que ellos hacen parte de las interacciones que se presentan en la naturaleza y, a partir del trabajo realizado con mariposas, describen algunas de las situaciones que emergen de las interacciones; por ejemplo, comportamientos adaptativos que mejoran el éxito reproductivo (Kricher, 2010).

Además, identifican características de las estrategias $\mathrm{R}$ y K, ejemplificándolas en otras especies y elaborando diagramas de pensamiento para establecer relaciones entre tipos de interacciones ecológicas y curvas de supervivencia.

\section{Instrumento 3. Ejercicio en clase preguntas desestabilizadoras}

\begin{tabular}{|c|c|c|}
\hline $\begin{array}{c}\text { Descriptor } \\
\text { (preguntas orientadoras) }\end{array}$ & Evidencia & Tendencia \\
\hline $\begin{array}{l}\text { 1) ¿Por qué cree usted que pueden } \\
\text { estar desapareciendo las abejas? }\end{array}$ & $\begin{array}{l}\text { - "Los humanos las consideran peligrosas, por lo que exterminan } \\
\text { - "La contaminación en el aire y ciertos químicos tal vez están } \\
\text { desorientando a las abejas, por lo que mueren sin volver al } \\
\text { panal y reduce la tasa de alimentación de las demás" (E1) } \\
\text { - "Las señales wifi y de los celulares afectan los sistemas de } \\
\text { ubicación de las abejas así que salen del panal pero no pueden } \\
\text { volver (serie de televisión } 31 \text { minutos)" (E13) } \\
\text { - "Postulo dos hipótesis para este fenómeno 1) en controles de } \\
\text { plagas 2) es la alteración del paisaje" (E12) } \\
\text { - "Las razones por las que un insecto se extingue pueden expresar } \\
\text { un control de plagas que salió de control" (E10) } \\
\text { - "Una posible causa de la extinción de abejas es la destrucción } \\
\text { constante de sus paneles (la tala masiva de árboles) y la emisión } \\
\text { de gases tóxicos derivados de la industria pesada que llevados } \\
\text { por el viento pueden afectar comunidades lejanas de abejas" } \\
\text { (E7) }\end{array}$ & $\begin{array}{l}\text { Se asocia a la extinción por causas } \\
\text { antropogénicas. }\end{array}$ \\
\hline $\begin{array}{l}\text { 2) ¿Qué consecuencias traería la } \\
\text { extinción de estos organismos } \\
\text { para la ecología? }\end{array}$ & $\begin{array}{l}\text { - "La extinción de abejas afectaría considerablemente la } \\
\text { polinización de las plantas que dependen de ellas y por tanto la } \\
\text { extinción de estas mismas. Si bien esto no implica la extinción } \\
\text { de todas las plantas pues algunas como las coníferas y los } \\
\text { helechos" (E7) } \\
\text { - "Se alterarían las cadenas tróficas de las que hacen parte, se } \\
\text { reduciría el proceso de polinización" (E5) } \\
\text { - "Como polinizadores, las abejas son muy importantes en el ciclo } \\
\text { de vida de las plantas con flores para reproducirse. Sin abejas a } \\
\text { menos que otro polinizador ocupe su lugar, muchas especies de } \\
\text { plantas no tendrían la siguiente generación" (E1) } \\
\text { "Las consecuencias de la reducción poblacional de abejas } \\
\text { traería consigo una reducción de la biodiversidad de fauna } \\
\text { y flora a escala macro. Pues al ser consumidoras de néctar } \\
\text { y fomentar la polinización de las plantas las abejas son un } \\
\text { eslabón clave del desarrollo de las poblaciones de todas } \\
\text { aquellas especies que dependen de ella" }\end{array}$ & $\begin{array}{l}\text { El estudiante reconoce } \\
\text { implicaciones en la red trófica al } \\
\text { desaparecer una especie. }\end{array}$ \\
\hline
\end{tabular}


A partir de las respuestas se observan dos tendencias principalmente: se asocia la extinción por causas antropogénicas, y el estudiante reconoce implicaciones en la red trófica al desaparecer una especie

Con respecto a este instrumento, es importante resaltar que el estudiante reconoce que el impacto humano genera alteraciones en las redes tróficas y estas actividades pueden afectar simultáneamente al entorno que nos rodea, relaciona las consecuencias con factores contaminantes y piensa en la extinción de la especie como única posibilidad a la problemática planteada. Frente a este último punto, autores como Bermudez y De Longhi (2008) lo ven como una dificultad en el aprendizaje de ecología, puesto que se configura desde la idea catastrófica de los acontecimientos, sin tener en cuenta que este puede ser un factor previo a la aparición de nuevas especies y que la extinción reconstruye los eventos que han ocurrido en el pasado y presente.

Por otro lado, la visión que muestra el estudiante recalca la idea de que los "problemas" de tipo ambiental tienen como única causa la contaminación; este tipo de miradas se asumen como una "percepción simplificadoraysesgada hacia los problemas de degradación", según Jiménez Aleixandre (2003), citado por Bermudez y De Longhi (2008).

\section{Instrumento 4. Re- aplicación de la prueba diagnostica}

\begin{tabular}{|c|c|c|}
\hline $\begin{array}{l}\text { Descriptor. } \\
\text { Preguntas de la encuesta }\end{array}$ & Evidencia & Tendencia \\
\hline \multirow[t]{4}{*}{$\begin{array}{l}\text { 1. Sobre las imágenes que se } \\
\text { muestran a continuación, explique } \\
\text { qué observa y con qué fenómeno } \\
\text { lo asocia }\end{array}$} & $\begin{array}{l}\text { - "La imagen parece representar un fenómeno de } \\
\text { depredación, que una especie ejecuta sobre otra. Es un } \\
\text { fenómeno natural que obedece a las necesidades biológicas } \\
\text { de las especies" (E4) } \\
\text { - "Hay un león comiendo un buey, es predación ya que } \\
\text { el buey es alimento del león y este lo está cazando para } \\
\text { sobrevivir" (E7) } \\
\text { - "Depredación león cazando búfalo" (E1) }\end{array}$ & $\begin{array}{l}\text { - Los estudiantes coinciden } \\
\text { en que el fenómeno que se } \\
\text { presenta es depredación, } \\
\text { en algunos casos usan la } \\
\text { descripción de la imagen. }\end{array}$ \\
\hline & $\begin{array}{l}\text { - "Depredación" (E1) } \\
\text { - "Fenómeno de alimentación vemos una hoja a medio } \\
\text { comer probablemente por una oruga" (E6) } \\
\text { - "Una hoja mordida por una oruga, es decir, sirvió de } \\
\text { alimento para esta, que se beneficia de los nutrientes de la } \\
\text { hoja" (E3) } \\
\text { - "Predación, una hormiga comiendo una hoja" (E11) } \\
\text { - "Es una hoja mordida. Al alimentarse sin matar la planta es } \\
\text { parasitismo" (E2) } \\
\text { - "Insecto se alimentan de una planta heterotrofia } \\
\text { parasitaria" (E13) }\end{array}$ & $\begin{array}{l}\text { - Depredación y alimentación. } \\
\text { - Parasitismo. }\end{array}$ \\
\hline & $\begin{array}{l}\text { - “Un parásito habita en un humano" (E13) } \\
\text { - "Parasitismo en humano"(E14) } \\
\text { - "En esta imagen se ve una hinchazón en el cuello de un } \\
\text { hombre causada seguramente por un parásito, como } \\
\text { pueden ser las larvas de una mosca" (E5) } \\
\text { - "En la imagen se muestra la herida que dejó sobre una } \\
\text { persona, las acciones de un parásito que se encontraba en } \\
\text { su interior" (E4) }\end{array}$ & - Parasitismo. \\
\hline & $\begin{array}{l}\text { - "Mutualismo la abeja poliniza y recoge miel" (E15) } \\
\text { - "Mutualismo: los dos se benefician la abeja obteniendo } \\
\text { néctar de las flores y regándoles polen (E8) } \\
\text { - "Aquí se puede ver la relación de mutualismo ya que esta } \\
\text { acción implica un beneficio para ambas partes" (E5) }\end{array}$ & - Mutualismo. \\
\hline
\end{tabular}




\begin{tabular}{|c|c|c|}
\hline $\begin{array}{l}\text { Descriptor. } \\
\text { Preguntas de la encuesta }\end{array}$ & Evidencia & Tendencia \\
\hline $\begin{array}{l}\text { 2. De las aves que se muestran a } \\
\text { continuación marque con una " } X \text { " } \\
\text { el grupo que considera tiene una } \\
\text { ventaja mayor, a la hora de obtener } \\
\text { su alimento, sobre las otras y } \\
\text { mencione por qué la selecciona. }\end{array}$ & $\begin{array}{l}\text { - "Ninguna, puesto que cada ave se alimenta y está adaptada } \\
\text { para una dieta diferente, cada pico sirve para frutas, } \\
\text { semillas, bichos etc." (E17) } \\
\text { - "Ninguna, cada una se especializa en un diferente alimento" } \\
\text { (E14) } \\
\text { "Creo que cada cual tendrá ventaja en un contexto } \\
\text { respectivo" (E11) } \\
\text { - "Estas aves tienen un pico que no está especializado para } \\
\text { determinada alimentación y permitiría a las aves conseguir } \\
\text { más alimentos diversos" (E1) } \\
\text { - "Hay muchas menos especies por lo que la competencia es } \\
\text { menor" (E9) } \\
\text {-Al ser pocos requieren de menos alimento para sobrevivir, } \\
\text { además, tienen distintos tipos de picos para alcanzar } \\
\text { alimentos" (E6) }\end{array}$ & $\begin{array}{l}\text { - Cada una tiene los picos de } \\
\text { acuerdo a sus requerimientos } \\
\text { alimenticios. } \\
\text { - Competencia. }\end{array}$ \\
\hline $\begin{array}{l}\text { 3. Indique qué entiende por } \\
\text { competencia ecológica y mencione } \\
\text { qué organismos relaciona más } \\
\text { fácilmente con el tema } \\
\text { 4. ¿En el lugar en el que usted } \\
\text { habita es posible ver interacciones } \\
\text { ecológicas? ¿Cómo las reconoce? } \\
\text { Argumente su respuesta y brinde } \\
\text { algunos ejemplos. }\end{array}$ & $\begin{array}{l}\text { - "Cuando dos especies o individuos compiten por los } \\
\text { mismos recursos, por ejemplo, manadas de suricatos } \\
\text { compitiendo por terreno, comida, hembras" (E14) } \\
\text { - "competencia ecológica es cuando dos o más compiten con } \\
\text { los recursos de un ecosistema. Por ejemplo cuando diversos } \\
\text { herbívoros compiten por la mejor planta" (E2) } \\
\text { - "Cuando una especie compite con otra por beneficios como } \\
\text { alimento o territorio. Hienas, cocodrilos, peces" (E13) } \\
\text { - "En la competencia ecológica distintas especies desarrollan } \\
\text { distintos métodos para conseguir recursos" (E1) } \\
\text { - "Pues claro, a veces veo aves depredando lombrices, gatos } \\
\text { depredando ratones" (E13) } \\
\text { - "Sí, simplemente viendo los pájaros y bichos se pueden ver } \\
\text { diferentes interacciones, principalmente de depredación y } \\
\text { mutualismo para los insectos polinizadores" }\end{array}$ & $\begin{array}{l}\text { - Disputa entre dos organismos. } \\
\text { que ven diversas interacciones } \\
\text { ecológicas y las relacionan con } \\
\text { ejemplos cotidianos }\end{array}$ \\
\hline
\end{tabular}

Con respecto al instrumento de cierre del proyecto, cabe anotar que se hace generalizada la idea de depredación y se contempla esta interacción tanto de animal a animal como de animal a planta. Sin embargo, persisten ideas de parasitismo (confundiendo los dos fenómenos). A nivel general los estudiantes reconocen situaciones y predicen otras causas de lo que observan (por ejemplo la situación del moscardón en el cuello del hombre); reconocen la importancia de interacciones como la polinización, y la asocian con una interacción de tipo mutualista.

Emergen otras relaciones en la forma de picos y cuerpos de algunos organismos como las aves, y las asocian con hábitos alimenticios y adaptación de ambientes, usan términos como competencia, en cuanto factor importante de co-evolución.

Cabe anotar que pese a las diversas actividades que se presentan, los estudiantes siguen usando ejemplos foráneos para ejemplificar interacciones ecológicas, además suelen hacer alusiones a interacciones como un fenómeno que determina jerarquías y una ubicación en la red alimenticia. Por otro lado, y pese a que en el instrumento 3 algunos de ellos reconocen que hacen parte de las interacciones ecológicas, cuando la pregunta no los vincula directamente, dejan de lado esta relación.

Al respecto, conviene decir que frente a la zona de desarrollo real que se presenta en los resultados de la prueba diagnóstica, para algunos estudiantes fueron favorables los ejemplos vistos en clase y fuentes de búsqueda externa. De esta manera, retomar sus ideas iniciales permite al formador tener un horizonte de trabajo y buscar ejercicios intencionados que permitan superar los errores iniciales. Así, "una investigación acerca de la evolución de estas concepciones permitirá sugerir situaciones y ayudas didácticas" (Giordan y De Vecchi, 1995, p. 245) que favorezcan el proceso de aprendizaje.

\section{Discusión}

Interpretar los procesos de modificabilidad de los estudiantes en la escuela, a través de la interacción y la construcción de estrategias didácticas que favorezcan la comprensión de los contenidos de enseñanza en 
torno a interacciones ecológicas, es un trabajo complejo, puesto que el ser humano es un sistema abierto y él mismo se encuentra actualmente permeado por diversas fuentes de información externa, como la televisión, internet, libros y vivencias propias. Estas fuentes externas hacen que sus estructuras de pensamiento se complejicen y puedan llegar a favorecer la comprensión de los temas que se abordan en la escuela o, por el contrario, puedan llegar a generar errores conceptuales y epistemológicos en la interpretación de un lenguaje tan complejo como el científico (Giordan y De Vecchi, 1995).

Vale la pena resaltar que algunas de las respuestas que se evidencian a lo largo del presente proyecto pueden dar cuenta y confirmar estudios preliminares que establecen errores epistemológicos y conceptuales en torno a los contenidos de enseñanza de ecología similares a los que se evidenciaron en el presente informe. Tal es el caso de la investigación realizada por Bermudez y De Longhi (2008), según la cual los estudiantes presentaban dificultades en el aprendizaje de conceptos estructurantes, como ecosistema, estabilidad y redes tróficas en estudiantes presentes en contextos educativos.

Por otro lado, el haber incluido ejemplos locales y experiencias concretas ( a través del ciclo de vida de Leptophobia aripa,), para superar lo que se ha conocido como la "extinción de la experiencia" (Pyle, 1993; Miller, 2005, citado por Bermudez y De Longhi, 2008), permitió que los estudiantes tuvieran la oportunidad de acercarse a una experiencia real contemplando la complejidad y belleza de una de las especies locales, de esta manera, quienes llevaron el ciclo de vida completo lograban predecir eventos y dar explicaciones desde los temas abordados en clase. En este caso, a través de este proyecto, se puede reiterar que el uso de ejemplos locales y la implicación de fenómenos que pueden ser observables por parte de estudiantes amplían su panorama y las estructuras mentales que le permiten comprender su realidad. De esta manera, como apunta Giordan y De Vecchi, "las confrontaciones con los hechos, y los discursos que se desprenden, permiten pues, de modo progresivo motivaciones externas o una serie de actividades adicionales por parte del mediador, para generar la reestructuración del concepto que ya han apropiado" (1995, p. 64)

\section{Conclusiones}

Los procesos de aprendizaje son muy com $\backslash$ plejos y se consolidan a lo largo de toda la vida de los sujetos, de esta manera el rompimiento de preconceptos o ideas "erradas" no puede darse de un momento para otro y el maestro como mediador en el aprendizaje debe partir de estas ideas previas para favorecer aprendizajes significativos y el pensamiento crítico en sus estudiantes.

Del mismo modo, debemos reconocer el contexto actual en el que nos desarrollamos, de esta manera no podemos ser ajenos a las fuentes de información alternativa, que también tienen lugar en la educación de nuestros estudiantes. Medios de comunicación como la internet, televisión o libros de texto, favorecen, o por el contrario, propician errores de tipo conceptual y epistemológico en temas como interacciones ecológicas. Por esta misma razón, el maestro debe reconocer estos errores para hacerles frente y buscar estrategias didácticas que permitan superar gradualmente preconceptos que generan dificultades en el aprendizaje. En esta misma línea, vale la pena reflexionar acerca de los ejemplos que se usan como referente, debido a que en su mayoría son ausentes los ejemplos locales y priman en la enseñanza el uso de documentales que muestran lo que sucede en las sabanas africanas o el mar.

A modo cierre, el presente informe permite reconocer la importancia de incluir contenidos de enseñanza en torno a interacciones ecológicas y evolución, debido a que esta visión permite que el estudiante reconozca que las otras especies representan un valor intrínseco dentro de la trama de la vida, además de que sin muchas de ellas las funciones vitales de los seres humanos cesarían o se verían seriamente afectadas. Se entiende, pues, la necesidad de cambiar la relación actual del hombre con su ambiente, y propiciar actitudes de cuidado y conservación de las especies con las que coexiste.

\section{Agradecimientos}

En primera medida, al Instituto Alberto Merani, por abrir sus puertas para hacer de este proyecto una realidad, por la acogida de cada una de las valiosas personas que con sus aportes y participación nutrieron mi formación y me han permitido enamorarme aún más de la profesión docente, en especial a la profesora Laura de Zubiría; quien hizo de mi paso por la escuela, la experiencia más maravillosa y gratificante.

Me siento completamente honrada de que este proyecto se hubiese convertido en el punto de partida para reconocer la importancia y el impacto que tiene el profesional de la educación para el país. Aprovecho para hacer una mención especial al profesor Julián de Zubiría y a la profesora Bertha Sarmiento, de mi parte, 
reciban mis respetos por establecer relaciones cada vez más amplias.

Agradezco a los estudiantes que me permitieron sorprenderme con cada una de sus preguntas y aportes, por mostrar interés en algo que personalmente me apasiona y le da sentido a mi existencia. Por último, pero no por eso de menor importancia, agradezco a la profesora Martha García y la profesora Sonia Martínez, quienes asesoraron mi trabajo y le aportaron desde su experiencia.

\section{Referencias}

Andrade, G., Henao, E., y Triviño, P. (Septiembre, 2013). Técnicas y procesamiento para la recolección, preservación y montaje de mariposas en estudios de biodiversidad y conservación. (Lepidoptera: hesperoidea- papilionoidea). Revista de la Academia Colombiana de Ciencias Exactas, Físicas y Naturales, 37(144), 311-325.

Arango, N., Elfi Chaves, M., y Feinsinger, P. (9 de noviembre de 2014). Guía metodológica para la enseñanza de la ecología en el patio de la escuela. Obtenido de: http://www.senderodechile.cl/wp-

Bermudez, G., y De Longhi, A. (2008). La Educación Ambiental y la Ecología como ciencia. Una discusión necesaria para la enseñanza. Enseñanza de las Ciencias, 7(2), 275-295.

Bonilla, E y Rodríguez, P. (1997). Más allá del dilema de los métodos. Bogotá: Grupo editorial Norma, Universidad de los Andes.

Castro, J. (2015). El papel de la Filosofía de la Biología en la formación de profesores de Biología. Esbozo de algunas propuestas, Los Retos de la Filosofía de la Biología en Iberoamérica. II congreso de la asociación iberoamericana de filosofía de la biología. Valle de bravo: México.

Chávez, M (1986). Aplicación del análisis de contenido a la entrevista. Ciencias Sociales, $\mathrm{n}^{\circ}$. 33. Universidad de Costa Rica, pp. 135143

Dobzhanshy, T., Ayala, F., Stebbins, G., y Valentine, J. (1970). Evolución. Davis, CA: Omega.

Feuerstein, R. (11 de octubre de 2010). Teoría de la modificabilidad estructural cognitiva y el papel del mediador. Obtenido de: http://www.utemvirtual.cl/plataforma/aulavirtual/assets/asigid_745/contenidos_arc/39250_c_feuerstein.pdf

Giordan, A., y De Vecchi, G. (1995). Los orígenes del saber. Sevilla: Diada.

Hernández, R., Fernández, C y Baptista, P. (2006). Metodología de la investigación.México D.F, México: McGraw-Hill Interamericana.

http://educacionquimica.info/include/downloadfile. php?pdf=pdf819.pdf

Kricher, J. (2010). Un compañero neotropical (Trad. I. Jaramillo, A y Segura, L). Colorado: Princeton University.

Longhi de, A. (2000). El discurso del profesor y del alumno: análisis didáctico en la clase de ciencias. Investigación didáctica, 18(2),201-216. Tardif, M. (2004). Los saberes del docente y su desarrollo profesional. Ciudad: CórdobaArgentina

Matthews, M. (1994). Historia, filosofia y enseñanza de las Ciencias: La aproximación actual. Enseñanza de Las Ciencias, 12, 255-277.

Tebar, L. (2009). El profesor mediador del aprendizaje. En L. Tebar, El profesor mediador del aprendizaje (p. 20). Bogotá: Cooperativa Magisterio.

Torres, R I. (2013). Como elaborar un informe publicable acerca del ciclo de vida de un lepidóptero. Museo de Historia Natural. UniversidadPedagógica Nacional.

Torres, R y García, M (2011). Taller Mariposas para Educar. Bio-Grafía Escritos sobre la Biología y su Enseñanza. ISSN 2027 1034. P. p. 512- 514.

Zubiría, J. (2006). Los modelos pedagógicos hacia una pedagogía Dialogante [2. ${ }^{a}$ edición]. Bogotá: Magisterio.

Zubiría de, J., Andrade, G., Sarmiento, B., Marin, J., Vargas, V., y Sampedro, H. (2009). Los ciclos en educación. Bogotá: MaMatthews, M. (1994). Historia, filosofia y enseñanza de las Ciencias: La aproximación actual. Enseñanza de Las Ciencias, 12, 255-277. 